\title{
Developed artificial neural network based human face recognition
}

\author{
Maryam Mahmood Hussein ${ }^{1}$, Ammar Hussein Mutlag², Hussain Shareef ${ }^{3}$ \\ ${ }^{1,2}$ Department of Computer Engineering Techniques, Electrical Engineering Technical College, Iraq \\ ${ }^{3}$ Department of Electrical Engineering, College of Engineering, United Arab Emirates University, United Arab Emirates
}

\begin{tabular}{|c|c|}
\hline Article Info & ABSTRACT \\
\hline Article history: & \multirow{9}{*}{$\begin{array}{l}\text { Face recognition has become one of the most important challenging } \\
\text { problems in personal computer-human interaction, video observation, } \\
\text { and biometric. Many algorithms have been developed in recent years. } \\
\text { These algorithms are not sufficiently robust to address the complex images. } \\
\text { Therefore, this paper proposes a soft computing algorithm based on face } \\
\text { recognition. One of the most promising soft computing algorithms which is } \\
\text { back-propagation artificial neural network (BP-ANN) has been proposed. } \\
\text { The proposed BP-ANN has been developed to improve the performance of } \\
\text { face recognition. The implementation of the developed BP-ANN has been } \\
\text { achieved using the MATLAB environment. The developed BP-ANN requires } \\
\text { supervised training to learn how to anticipate results from the desired data. } \\
\text { The BP-ANN has been developed to recognize } 10 \text { persons. Ten images have } \\
\text { been used for each person. Therefore, } 100 \text { images have been utilized to train } \\
\text { the developed BP-ANN. In this research } 50 \text { images have been used for } \\
\text { testing purpose. The results show that the developed BP-ANN has produced } \\
\text { a success ratio of } 82 \% \text {. }\end{array}$} \\
\hline Received Feb 16, 2019 & \\
\hline Revised May 25, 2019 & \\
\hline Accepted Jun 28, 2019 & \\
\hline Keywords: & \\
\hline $\begin{array}{l}\text { Back-propagation artificial } \\
\text { neural network (BP-ANN) }\end{array}$ & \\
\hline Face detection & \\
\hline Face recognition & \\
\hline Viola-Jones algorithm & \\
\hline
\end{tabular}

Copyright $\odot 2019$ Institute of Advanced Engineering and Science. All rights reserved.

\section{Corresponding Author:}

Maryam Mahmood Hussein,

Department of Computer Engineering Techniques,

Electrical Engineering Technical College,

Middle Technical University, Baghdad, Iraq.

Email: maryammahmood202@gmail.com

\section{INTRODUCTION}

In the present networked world, the need to keep up the security of data and physical property is becoming increasingly essential and increasingly difficult. From time to time we find out about the crimes of credit cards extortion, breaking of the personal computer by hackers, or on the other hand security breaks in an organization or government building. Recently, technology becomes accessible to permit verification of genuine individual identity. This technology depends on a field called biometrics. Face recognition represents one of the few biometric techniques [1]. It aims to imitate one of the natural human capacities: perceiving characteristics of faces in different situations and connect them with the learning stored in their memory. It is a simple and direct task for peoples, however, it seems to be a challenging problem for computing systems because of the few natural factors such as face location, lighting condition, and the distance of face from the camera [2].

Many soft computing techniques have been mentioned in the literature [3-6]. A new discriminant face recognition methodology, which is based on principal component analysis algorithm (PCA) and adaptive neuro-fuzzy inference system (ANFIS) has been proposed in [7]. PCA has been used to extract the feature of facial image and decrease its dimensions; meanwhile, ANFIS has been used as a classifier for face recognition. However, the ANFIS is required a very long time for training. In [8], a face recognition system has been implemented based on random forest (RF) classifier and histograms of oriented gradients (HOG) 
face descriptor. However, the increasing of the number of trees in the random forest will make the system slower. Meanwhile, support vector machine (SVM) based on chaotic particle swarm optimization (CPSO) algorithm using improved circle map has been implemented in [9]. However, trap in local minima is the main drawback of the particle swarm optimization.

Therefore, to overcome these drawbacks one of the most algorithms of pattern recognition namely back-propagation artificial neural network (BP-ANN) has been used in this research to implement the face recognition system. The paper is organized as follows: Section 2 describes design of the proposed system. Viola-Jones algorithm has been presented in Section 3. Section 4 explains the back-propagation artificial neural network. The implementation of the proposed system is found in Section 5. The discussion of the results is presented in Section 6. Finally, the conclusion is drawn in Section 7.

\section{DESIGN OF THE PROPOSED SYSTEM}

The proposed human face recognition system is shown in Figure 1. It passes in five steps which are image acquisition, face detection, feature extraction, face recognition, and recognition report. Image acquisition phase represents the first stage of a face recognition system. Therefore, the user gives the face image as the input to the system. The next step required for facial recognition system is face detection. It is the process of deciding whether a sub-window of an input image contains a face or not. In this research, the detection of the face has been implemented using the Viola-Jones algorithm. The third phase is feature extraction from the face image. Principal component analysis (PCA) algorithm has been used to extract these features. It is one of the most famous algorithms of features extraction [10]. Regarding the fourth phase is face recognition. In this module, the system takes the face of an individual to be recognized and compare it with the faces that already trained in the neural network and it recognized the best matching face as output. For this purpose back-propagation artificial neural network (BP-ANN) algorithm has been used. Finally, the system classified the input face image as known or unknown person.

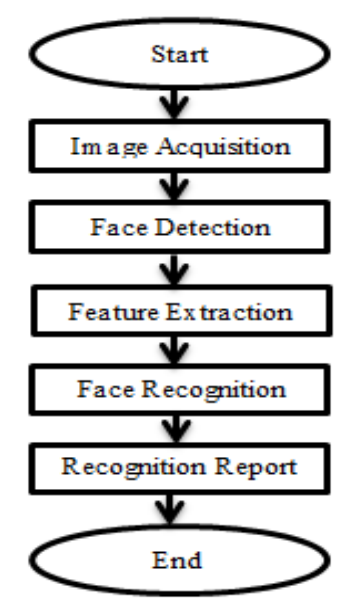

Figure 1. The proposed human Face recognition system

\section{VIOLA-JONES ALGORITHM}

Viola-Jones algorithm is one of the most important and famous methods have been used to detect the human face from an entire image [11]. It is proposed in the year 2001 by Paul Viola and Michael Jones. The goal of this algorithm is the issue of face detection but can also be implemented for detecting diverse objects such as eyes, nose, and mouth [12]. This method contains four basic components as described in the following:

\subsection{Harr Features}

The essential part of this process is the extraction of Harr features from the image using Harr cascade classifier. Each feature restores a single value which is given by subtracting the sum of the pixels in the white area from the sum of pixels in the black area [13], as defined below:

$$
\mathrm{F}(\mathrm{x})=\Sigma \text { (pixels in black area) }-\Sigma \text { (pixels in white area) }
$$




\subsection{Integral Image}

To reduce the computational time, another image representation has been used called integral image [14]. The value of each $(x, y)$ location in the integral image is the sum of pixels above and left of this location at the original image [15].

\subsection{AdaBoost Algorithm}

Viola-Jones algorithm has been used a $24 \times 24$ window size as the base window to begin the extraction process of Harr features from any given image [15]. Thus, around 160000 features can be extracted by using this window which is practically impossible [13]. For this reason, the AdaBoost algorithm has been used to find the best features among 160000. These features represent the weak classifiers. The AdaBoost algorithm creates strong classifiers as a linear combination of these weighted weak classifiers as shown below [15]:

$$
F(x)=a_{1} f_{1}(x)+a_{2} f_{2}(x)+a_{3} f_{3}(x)+\ldots
$$

Where $F(x)$ represents the strong classifier, while $f_{1}(x), f_{2}(x)$ and so on represents weak classifiers, a $a_{1}, a_{2}$ and so on are weighted quantities and $\mathrm{x}$ is an image [16].

\subsection{Cascade Classifier}

Cascade classifier is a method for combining strong classifiers created by AdaBoost algorithm in cascade stages. These stages have been used to determine whether the sub-window for the given image is a face or non-face [17].

\section{BACK-PROPAGATION ARTIFICIAL NEURAL NETWORK}

Artificial neural network (ANN) is an information processing tool that is inspired by the way the human brain work principle [18]. It is composed of a large number of interconnected processing neurons. Back-propagation artificial neural network (BP-ANN) represents one of the most commonly used families of neural networks for pattern recognition due to its relative simplicity [19]. It based on gradient descent to update the weights in order to reduce the squared error between the output and target values of the network [20]. BP-ANN is a multilayer feedforward, supervised learning network. It goes in both directions forward and backward. In the forward direction, training data is fed to the neural network through the input layer. Then data is applied to the hidden layer, the processing of data is actually performed at the hidden layer. Then, the data is applied to the output layer. The output calculated in the forward direction is compared with the target output. The difference between the target output and the calculated output provide the error. This error is calculated and propagated back towards the lower layer of the network for updating the weights of each layer to minimize it [21]. This error is given by the mean square error (MSE) as defined below:

$$
\operatorname{MSE}=\frac{1}{n} \sum_{i=1}^{n}\left(T_{i}-Y_{i}\right)^{2}
$$

Where $T_{i}$ is the target output of the network, $Y_{i}$ represent the calculated output, and $\mathrm{n}$ is the number of the network samples [22].

\section{IMPLEMENTATION OF THE PROPOSED SYSTEM}

The system for human face recognition involves four stages. These stages are the pre-processing stage, training stage, implementation of back-propagation artificial neural network and testing stage. These stages will describe in the next sections.

\subsection{Pre-processing Stage}

In this stage, the Viola-Jones algorithm has been used to detect the face region from an entire image, which is referred to as a face detection process. Then, the PCA algorithm has been used to extract the main important features from the detected face image. Therefore, in this work, each face image is represented by 100x1 feature vector. Here, databases of 100 face images have been used with different faces of 10 persons. As a result, 100x100 database has been used for training the neural network. A robust and efficient face recognition system should be insensitive to noise generated by cameras or frame grabbers. For this reason, it is very important to add noise to some image face samples for each person that already been stored in the face database. 


\subsection{Training Stage}

As described earlier during the pre-processing stage, the feature vectors for face images are computed to train the neural network. MATLAB environment has been used to implement a physical feed forward back-propagation neural network.

\subsection{Implementation of Back-Propagation Artificial Neural Network}

The proposed architecture of BP-ANN model for proposed face recognition system is shown in Figure 2. It contains four layers; input layer, two hidden layers, and output layer.

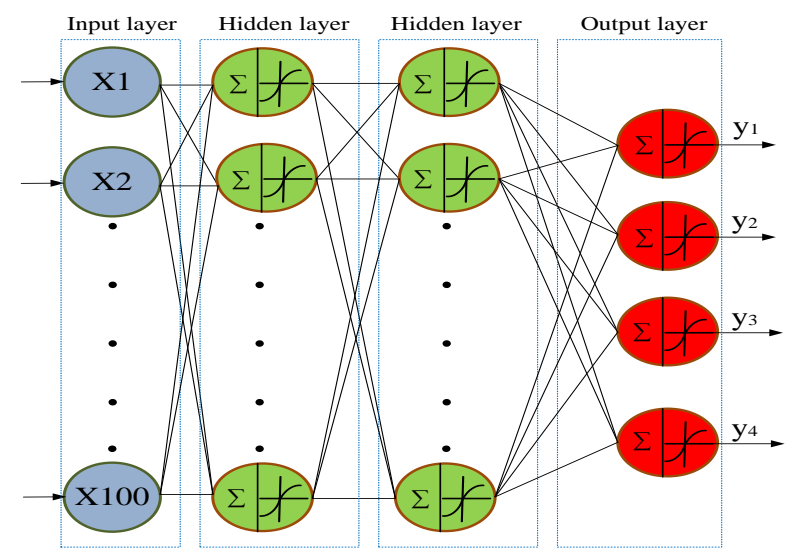

Figure 2.The architecture of the develop ANN

The input layer represents the image of the face as a system input. Therefore, the number of neurons in the input layer depends on the dimensions of an image which in this work is equal to 100 neurons. In the artificial neural network, two variables have been considered namely learning rate and the number of hidden neurons. The range value of the learning rate is greater than 0 and equal to or less than 1 . Three values of the learning rate which are $0.2,0.4$, and 0.8 have been tested in the training of ANN to determine the appropriate learning rate value. The ANN with a learning rate of 0.4 gives the most accurate results. Regarding the hidden layer, the ANN was trained using 25, 55, and 85 neurons. The appropriate neurons have been found to be 55 neurons for both hidden layers. The hidden neurons perform the calculation using bipolar sigmoid activation function as defined below:

$$
\begin{aligned}
& Z_{\text {inj }}=\left[\sum_{i=1}^{100} \mathrm{x}_{i} w_{i j}\right]+b_{j} \text { where } \mathrm{j}=1,2, \ldots \ldots, 55 \\
& Z_{j}=\frac{2}{1+e^{-Z_{\text {inj }}}}-1 \text { where } \mathrm{j}=1,2, \ldots \ldots, 55
\end{aligned}
$$

The last layer is the output layer. The number of neurons in the output layer depends on the nature of the problem. The developed system has been proposed for 10 persons. To decrease the neurons in the output layer, a look up table has been used. Therefore, only four neurons have been used in the output layer. The look up table is shown in Table 1.

Table 1. Lookup Table for the Proposed Persons

\begin{tabular}{ccccc}
\hline Target Person & Y1 & Y2 & Y3 & Y4 \\
\hline Dima & 0 & 0 & 0 & 0 \\
Abdulazeez & 0 & 0 & 0 & 1 \\
Mohammed & 0 & 0 & 1 & 0 \\
Reem & 0 & 0 & 1 & 1 \\
Mostafa & 0 & 1 & 0 & 0 \\
Jumana & 0 & 1 & 0 & 1 \\
Teeba & 0 & 1 & 1 & 0 \\
Ibraheem & 0 & 1 & 1 & 1 \\
Rania & 1 & 0 & 0 & 0 \\
Hala & 1 & 0 & 0 & 1 \\
\hline
\end{tabular}

Indonesian J Elec Eng \& Comp Sci, Vol. 16, No. 3, December 2019 : 1279 - 1285 
The bipolar sigmoid activation faction has been used to perform the calculation in the output layer as defined below:

$$
\begin{aligned}
& Y_{\text {ink }}=\left[\sum_{j=1}^{55} \mathrm{z}_{j} v_{j k}\right]+b_{k} \text { where } \mathrm{k}=1,2,3,4 \\
& Y_{k}=\frac{2}{1+e^{-Y_{\text {ink }}}}-1 \text { where } \mathrm{k}=1,2,3,4
\end{aligned}
$$

\subsection{Testing Stage}

This stage is used to evaluate the performance of the proposed face recognition system. In this research 50 images have been used for testing purpose. The selected testing image is given to the trained neural network. Then, this image is compared with the face images of the trained neural network. Therefore, it recognized the best matching face as output.

\section{RESULTS AND DISCUSSION}

In this paper, a human face recognition system has been implemented. The face recognition system consisted of two main parts. The first part is the detection process of the face from an entire image. This purpose has been achieved using the most important face detection method called the Viola-Jones algorithm. The second part represents the recognition of the face. BP-ANN has been implemented for this purpose. In the training of ANN, two parameters must be adjusted, namely, learning rate and the number of neurons in the hidden layer. The appropriate value of the learning rate is 0.4 ; meanwhile, the appropriate value of neurons in both hidden layers are 55 neurons. The MSE values are then averaged from the training results. Figure 3 shows the number of epochs against the MSE which is 1.4143E-5. After the ANN is trained, it can be used for on-line human face recognition.

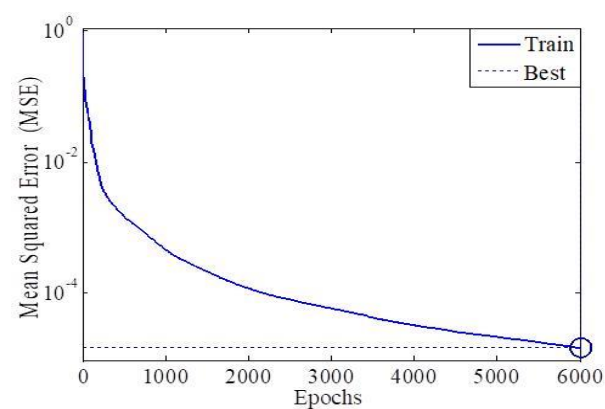

(a)

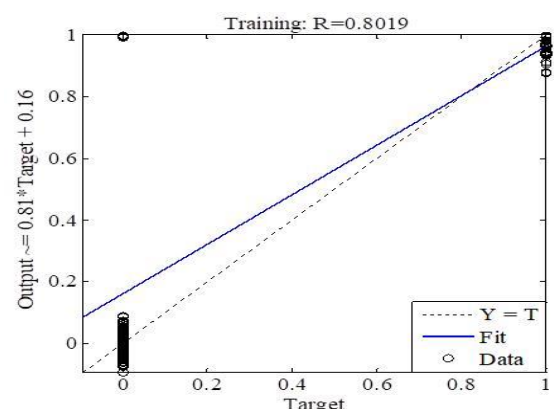

(b)

Figure 3. (a) Performance curve with respect to MSE, (b) Regression plot of training data

The detection and recognition process has been achieved using the graphical user interface (GUI) of the MATLAB environment. 50 images have been subjected for testing purpose. BP-ANN succeeded in recognized 41 images. Meanwhile, failed to recognized 9 images. The sample of the recognized image is shown in Figure 4.

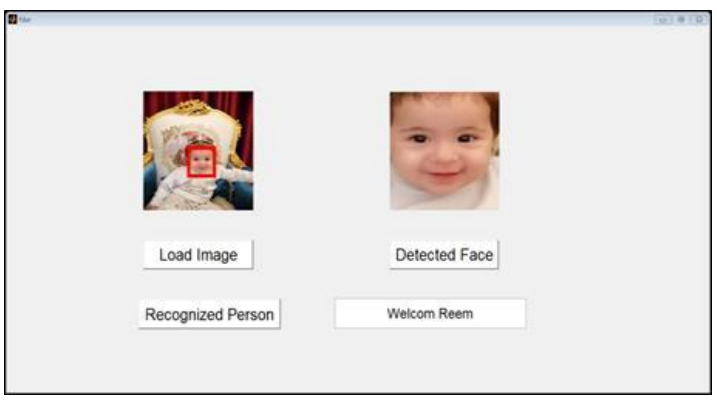

Figure 4. Sample of face recognition using neural network 
The ANN has been subjected to 50 images as mentioned before. Some of these images are not in the ANN database. Therefore, the ANN succeed to recognize these images. Sample of the not recognize persons is shown in Figure 5.

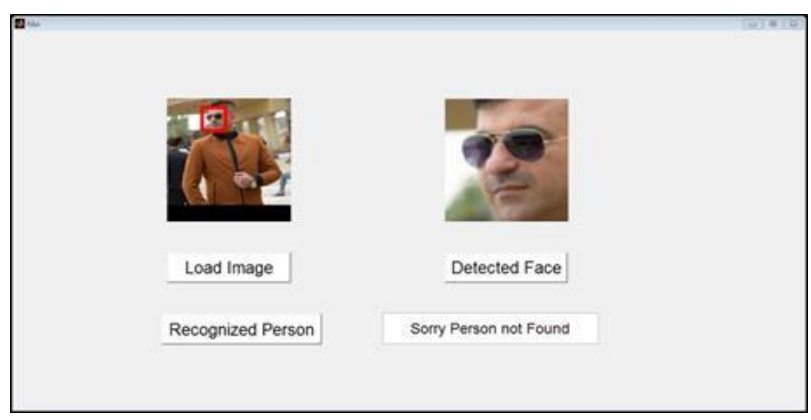

Figure 5. Sample of face not recognition using neural network

After all images were subjected to ANN, the success recognition ratio has been found to be $82 \%$. Meanwhile, the failed ratio has been found to be $18 \%$. The graphical representation of results can be shown in Figure 6. The figure shows that the ANN has been achieved acceptable results. Therefore, the ANN can be used for face recognition.

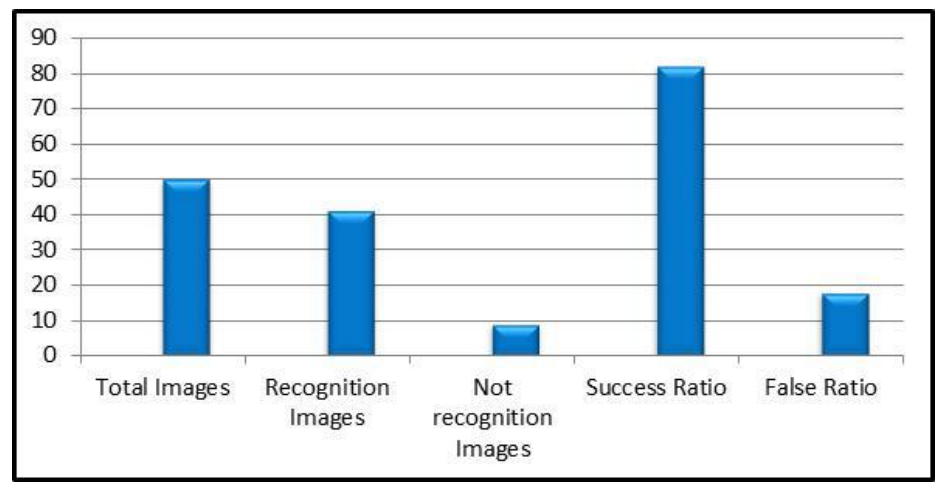

Figure 6. Performance representation of back-propagation neural network

\section{CONCLUSION}

Today, face recognition system play an important role in many applications such as authentication, indexing, monitoring, and other surveillance applications. In this paper, one of the most common soft computing algorithms which is the BP-ANN has been proposed for face recognition. The appropriate values of the learning rate and neurons in the two hidden layers have been found to be $0.4,55$, and 55 respectively. Therefore, the ANN has been developed with 100-55-55-4 architecture. The performance evaluation has been achieved under the MATLAB environment. 50 images have been used for testing purpose. The ANN succeeds to recognize 41 images and fail to recognize 9 images. Therefore, the success ratio is $82 \%$ meanwhile; the failed ratio is $18 \%$. Through testing results of the experiment, this system achieves a higher recognition rate. Therefore, good results can be achieved using the developed BP-ANN. Furthermore, the BPANN algorithm is fast and relative simple to implement.

\section{REFERENCES}

[1] S. Mantri, K. Bapat, "Neural network based face recognition using Matlab", International Journal of Computer Science and Engineering Technology, vol. 1, 2011, pp. 6-9.

[2] G. Ramkumar, E. Logashanmugam, "Multimodal verge for scale and pose variant real time face tracking and recognition”, Indonesian Journal of Electrical Engineering and Computer Science, vol. 13, 2019, pp. 665-670. 
[3] A. H. Mutlag, H. Shareef, A. Mohamed, "Design and analysis of a high performance three-phase voltage source inverter based on fuzzy logic controller", Student Conference on Research and Development, IEEE, SCOReD 2013.

[4] H. Shareef, A. H. Mutlag, A. Mohamed, "Random Forest-Based Approach for Maximum Power Point Tracking of Photovoltaic Systems Operating under Actual Environmental Conditions", Computational Intelligence and Neuroscience, vol. 2017, Article ID 1673864, 17 pages, 2017. https://doi.org/10.1155/2017/1673864.

[5] H. Shareef, M. M. Islam, A. A. Ibrahim, A. H. Mutlag, "A nature inspired heuristic optimization algorithm based on lightning", International Conference on Artificial Intelligence, Modelling and Simulation, 2015.

[6] Mujiarto, A. Djohar, et al. " Colored object detection using 5 dof robot arm based adaptive neuro-fuzzy method", Indonesian Journal of Electrical Engineering and Computer Science, vol. 13, 2019, pp. 293-299.

[7] T. Chandrasekhar, Ch. Sumanth," Face recognition system using adaptive neuro-fuzzy inference system”, International Conference on Electrical, Electronics, Communication, Computer and optimization Techniques, IEEE, 2017.

[8] A. Salhi, M. Kardouchi, N. Belacel, "Fast and efficient face recognition system using random forest and histograms of oriented gradients", International Conference of the Biometrics Special Interest Group, 2012.

[9] M. Li, X. Sun, Y. Wang, W. Li, Y. Hao, "Application of improved CPSO-SVM approach in face recognition", International Conference on Artificial Intelligence and Computational Intelligence, IEEE, 2009.

[10] N. S. Parameswaran, D. Venkataraman, "A computer vision based image processing system for depression detection among students for counseling", Indonesian Journal of Electrical Engineering and Computer Science, vol. 14, 2019, pp. 503-512.

[11] N. Deshpande, S. Ravishankar, "Face detection and recognition using Viola-Jones algorithm and fusion of PCA and ANN", Advances in Computational Sciences and Technology, vol. 10, 2017, pp.1173-1189.

[12] S. Tikoo, N. Malik, "Detection of face using Viola Jones and recognition using back propagation neural network", Department of Electrical and Electronics and Communication Engineering, The North Cap University, Gurgaon, 2017.

[13] M. Nehru, P. S, “Illumination invariant face detection using Viola Jones algorithm”, International Conference on Advanced Computing and Communication Systems, IEEE, 2017.

[14] P. Viola, M. Jones, "Rapid object detection using a boosted cascade of simple features", Computer Society Conference, IEEE, 2001.

[15] A. Gupta, R. Tiwari, "Face detection using modified Viola Jones algorithm", International Journal of Recent Research in Mathematics Computer Science and Information Technology, vol. 1, 2014, pp. 59-66.

[16] P. Mehta, P. Tomar, "An efficient attendance management system based on face recognition using Matlab and raspberry pi 2", International Journal of Engineering Technology Science and Research, vol. 3,2016, pp.71-78.

[17] M. Dabhi, B. Pancholi, "Face detection system based on Viola-Jones algorithm", International Journal of Science and Research, vol. 5, 2016, pp. 62-64.

[18] R. A. Dilruba, N. Chowdhury, et al. "Data pattern recognition using neural network with Back-Propagation training”, International Conference on Electrical and Computer Engineering, IEEE, 2006.

[19] N. Belghini, A. Zarghili, et al. "A color facial authintification system based on semi supervised backpropagation neural network", International Conference in Multimedia Computing and systems, IEEE, 2011.

[20] D. Dolkar, B. Saha, "Optimal face recognition method using Ant Colony based Back-Propagation network", International Conference on Computer and Devices for Communication, 2009.

[21] T. Ahmad, A. Jameel, B. Ahmad, "Pattern recognition using statistical and neural techniques", International Conference on Computer Networks and Information Technology, IEEE, 2011.

[22] A. Suliman, Y. Zhang, "A review on Back-Propagation neural networks in the application of remote sensing image classification”, Journal of Earth Science and Engineering, vol. 5, 2015, pp.52-65. 